\title{
Política Industrial, Tecno-nacionalismo e Indústria 4.0: a Guerra Tecnológica entre China e EUA
}

Antônio Carlos Diegues (Instituto de

Economia, Unicamp)

José Eduardo Roselino (UFSCar)

Resumo: Este artigo analisa a evolução da guerra tecnológica entre China e EUA em um contexto de exacerbamento das estratégias tecno-nacionalistas de desenvolvimento industrial e tecnológico. Parte-se da hipótese de que tais políticas, ao impulsionarem as tecnologias características da Indústria 4.0, têm como objetivo forjar um novo paradigma tecno-econômico e reconfigurar as bases sobres as quais se assentam a dinâmica da concorrência intercapitalista e interestatal. A principal contribuição pretendida pelo trabalho é a análise das implicações da Guerra Tecnológica entre China e EUA a partir da segmentação do paradigma tecno-econômico da Indústria 4.0 em diferentes camadas, para além do debate tradicional acerca da transição para as redes $5 \mathrm{G}$.

palavras-chave: Guerra tecnológica; Tecno-nacionalismo; Industria 4.0; Política Industrial; Economia chinesa.

Código JEL: O53; O14; O25; O33; O38; L52.

Área Temática: 1.6 Novos temas - Indústria 4.0, Internet das coisas, outros 


\title{
Política Industrial, Tecno-nacionalismo e Indústria 4.0: a Guerra Tecnológica entre China e EUA
}

\author{
Industrial Policy, Techno-Nationalism and Industry 4.0: China-USA \\ Technological War
}

\begin{abstract}
This paper aims to analyze the evolution of the technological war between China and the USA in a context of exacerbation of techno-nationalist strategies for industrial and technological development. The hypothesis is that such policies, by boosting Industry 4.0, aim to create a new techno-economic paradigm and thus reconfigure the bases of intercapitalist and interstate competition. The main contribution to the literature is providing an analysis of the implications of the Technological War between China and the USA in different layers of the Industry 4.0 techno-economic paradigm, in addition to the mainstream debate about the transition to $5 \mathrm{G}$ communication networks.
\end{abstract}

Key-words: Technological war; Techno-nationalism; Industry 4.0; Industrial Policy; Chinese economy.

\section{Introdução}

Este artigo procura analisar a evolução da guerra tecnológica entre China e EUA a partir de uma perspectiva que relaciona tal movimento a três dimensões: (i) a retomada do debate sobre política industrial e desenvolvimento no cenário pós crise de 2008, (ii) o reflorescimento de estratégias tecno-nacionalistas de desenvolvimento, em um cenário de reavaliação de elementos que têm sustentado a dinâmica da globalização desde o último quartel do século XX, e (iii) a construção deliberada de esforços por parte dos países centrais no sentido de se empurrar a fronteira tecnológica em direção ao que se convencionou denominar de Indústria 4.0.

A hipótese é a de que a combinação destes elementos se insere em um contexto mais amplo que tem como objetivo forjar um novo paradigma tecnoeconômico e assim reconfigurar as bases sobres as quais se assenta a dinâmica da concorrência intercapitalista e interestatal. Essa reconfiguração, por sua vez, seria o elemento central para reafirmar a posição de liderança tecnológica e econômica das principais potências mundiais e ao mesmo tempo impor condicionantes ao avanço chinês nestas mesmas dimensões.

A partir deste arcabouço, são duas as contribuições pretendidas pelo trabalho. A primeira é apresentar um esforço de sistematização da política industrial chinesa, com ênfase no período pós publicação do Made in China 2025. Para tal, admite-se a coevolução entre as transformações nas políticas industriais e aquelas relacionadas à estratégia de desenvolvimento, bem como coexistência simultânea de diferentes estágios desta estratégia de desenvolvimento. Destaca-se que essa a coexistência combina, em diferentes regiões do país, setores, tipos de firmas e de tecnologias, políticas industriais qualitativamente distintas. Tal fato decorre de especificidades econômicas, geográficas e sociais que fazem com que o processo de desenvolvimento e catching-up chinês apresente natureza ímpar quando comparada às experiências dos gansos voadores. Ao contrário do que caracteriza a lógica dos gansos voadores, sugere-se que o avanço da estrutura produtiva chinesa em direção a áreas com maior complexidade tecnológica não implica necessariamente a redução absoluta da produção manufatureira doméstica em segmentos menos complexos e intensivos em mão de obra. Isso porque, ao invés de se internacionalizarem, tais segmentos seriam gradativamente deslocados internamente para regiões do território chinês com menores custos e menores níveis de desenvolvimento produtivo. 
A segunda e principal contribuição pretendida pelo artigo diz respeito à análise das implicações da Guerra Tecnológica entre China e EUA a partir da segmentação do paradigma tecnoeconômico da Indústria 4.0 em diferentes camadas, para além do debate tradicional acerca da transição para as redes de comunicação 5G. Assim, são analisados os potenciais desdobramentos desta disputa tecnológica nas camadas de sistemas cyber físicos em serviços e manufatura inteligentes, integração tecnológica (robotização, machine learning, impressão 3D) e infraestrutura tecnológica habilitadora (cloud computing, internet of things, sensorização e nova geração de semicondutores).

Conclui-se que as camadas nas quais as potencialidades de desenvolvimento chinesa parecem estar mais avançadas são aquelas relacionadas a serviços inteligentes, inteligência artificial e big data. Em contrapartida, é na sub camada de infraestrutura habilitadora relacionada aos semicondutores de última geração que se observa a lacuna central da estratégia chinesa expressa no Made in China 2025 e aos objetivos rascunhados para o China Standards 2035. Assim, dado o caráter altamente transversal e pervasivo destes, a superação dessa lacuna se coloca como a pedra angular para viabilizar o espraiamento das demais tecnologias a fim de se forjar um novo paradigma tecnoeconômico que reserve um espaço hierarquicamente superior ao parque produtivo e às tecnologias chinesas.

A fim de desenvolver tais contribuições, o artigo estrutura-se em duas seções, além desta introdução e das considerações finais.

\section{Política industrial como o vetor da estratégia desenvolvimento chinesa em direção à indústria 4.0}

Ao se analisar a relação entre a política industrial e a estratégia de desenvolvimento chinesa desde o último quartel do século XX, a primeira constatação é sua capacidade de reconfiguração. De maneira geral, um importante caracterizador deste movimento é coevolução entre as transformações nas políticas industriais e as demais dimensões relacionadas à estratégia de desenvolvimento (BRESSER-PEREIRA et al 2020, JABBOUR e de PAULA, 2020)1.

Além desta coevolução, em um plano lógico, destaca-se a existência simultânea de diferentes estágios da estratégia de desenvolvimento, que combinam em distintas regiões do país, setores e tecnologias, políticas industriais qualitativamente distintas. Tal fato decorre de especificidades econômicas, geográficas e sociais que conferem ao processo de desenvolvimento e catching-up chinês natureza histórica ímpar frente às demais experiências nacionais.

Dada a heterogeneidade estrutural produtiva, tecnológica, regional e social, somadas às dimensões geográficas e populacionais chinesas, este processo não pode ser compreendido como uma estratégia de emulação de experiências históricas de países como Japão e Coréia do Sul dentro de uma perspectiva da lógica dos gansos voadores (AKAMATSU, 1962 e PALMA, 2009). Isso porque a transformação estrutural voltada à construção de uma economia especializada em atividades de maior complexidade tecnológica, e que prescinda de manufaturas de média e baixa intensidade tecnológica, encontra limites óbvios para ser universalizada de modo a incorporar e estender os frutos do progresso técnico ao gigantesco contingente populacional chinês. A título de ilustração cabe enfatizar que os setores intensivos em trabalho e em recursos naturais representavam $38 \%$ do valor adicionado manufatureiro chinês em 2017 (INDSTAT2, UNIDO). No que diz respeito às exportações, apenas os setores de têxtil e vestuário foram responsáveis por um superávit de US\$ 365 bi em 2018, face a US\$ 383 bi dos setores de eletrônica e equipamentos elétricos.

Deste modo, ao mesmo tempo em que se observa a busca pela construção de uma economia orientada à inovação autóctone - caracterizada pelo Developed by China (WUBBEKE et al, 2016) -, é possível observar a coexistência dos pilares da política

\footnotetext{
${ }^{1}$ Os autores defendem a tese de que a coevolução entre os ciclos institucionais e as transformações na estratégia de desenvolvimento chinês é um instrumento distintivo da lógica de planejamento chinês, e é fundamental para se compreender sua dinâmica de transformação estrutural.
} 
industrial sobre os quais se sustentam a estratégia gestada desde o último quartel do século passado de se fomentar o movimento de Made in China - com presença até o período atual bastante sólida em setores de baixa intensidade tecnológica e altamente intensivos em mão de obra conforme defende Nolan (2013).

Em síntese, ao contrário do que sugeriria uma interpretação linear etapista do desenvolvimento chinês como uma emulação de modelos de outros países asiáticos a partir da lógica dos gansos voadores, nota-se a permanente complementariedade e dependência mútua entre os diversos estágios de desenvolvimento das forças produtivas domésticas.

Por um lado, essa complementaridade é necessária devido às ambições geopolíticas da civilização chinesa e ao desafio de se incorporar o enorme contingente demográfico chinês aos frutos do progresso técnico e a impossibilidade de fazê-lo a partir de um modelo export-led com uma inserção altamente especializada nas cadeias globais de valor. Por outro, só é possível dada a capacidade de coordenação de política industrial centralizada na National Development and Reform and Commission e na SASAC (State-owned Assets Supervision and Administration Comission) e à conseguinte socialização do investimento (BRESSER-PEREIRA et al 2020, JABBOUR e de PAULA, 2020).

Entende-se que as políticas industriais de fomento às atividades associadas ao que se tem convencionado denominar de Indústria 4.0 e à Economia Digital na China se inserem em um contexto mais amplo de transformação permanente da estrutura produtiva doméstica e do conseguinte padrão de inserção internacional.

Neste contexto, a estratégia expressa desde meados da década passada - por meio do Medium and Long Term Plan for the Development of Science and Technology e de seus megaprojetos - na busca pela construção de uma economia orientada à inovação autóctone teria dois grandes objetivos que coincidiriam com o duplo centenário chinês. Inicialmente, a busca seria pela consolidação de uma economia moderadamente próspera em 2021, no centenário da fundação do Partido Comunista Chinês. Com os esforços para se contornar a armadilha da renda média, os objetivos para 2049 - nas comemorações do centenário da Revolução Comunista - seriam de cristalização da economia chinesa como uma Superpotência Industrial e da Internet, com liderança mundial em setores de alta intensidade tecnológica (WUBBEKE et al, 2016).

Como desdobramento das iniciativas materializadas nas estratégias Made in China 2025 e Internet Plus, pretende-se contornar um duplo desafio. Por um lado, o acirramento das pressões competitivas derivadas das iniciativas dos países líderes para empurrar a fronteira tecnológica em direção à Industria 4.0 teria como objetivo reorganizar os determinantes da dinâmica concorrencial e suprimir os diferenciais competitivos construídos pela indústria chinesa. Por outro, a busca por reduções de custos a partir do permanente deslocamento de unidades produtivas em direção a outros países do leste e sudeste asiático também poderia impactar na redução da competitividade relativa chinesa principalmente em setores de média e baixa intensidade tecnológica.

A partir deste duplo enfrentamento, o fomento à Industria 4.0 e à Economia Digital seriam importantes vetores para viabilizar um incremento da capacidade de apropriação por parte das empresas chinesas nas cadeias globais de valor, contornando assim uma inserção hierarquicamente inferior.

O conjunto de políticas industriais e tecnológicas que pautam tal estratégia mais ampla se insere em um contexto de acirramento da competição intercapitalista internacional, que se acentua pós crise de 2008. A partir de uma intepretação que se convencionou denominar de tecno-nacionalista, tais políticas se organizam por meio de um eixo top down e se materializam em inúmeros esforços que se retroalimentam para reforçar o sistema nacional de inovação chinês em paralelo ao fortalecimento da estrutura produtiva (ZHOU e LIU, 2016 e CHEN e NAUGHTON, 2016).

Grosso modo, tais diretrizes podem ser compreendidas a partir da coexistência de três grandes vetores de materialização, os quais combinam esforços de $\mathrm{P} \& \mathrm{D}$, inovação, financiamento e formas de atuação do Estado de acordo com as estratégias e os atuais 
estágios de desenvolvimento produtivo e tecnológico da estrutura empresarial doméstica. De maneira esquemática - e com as limitações inerentes a quaisquer esforços de construção de uma tipologia -, é possível segmentar diferenças qualitativas importantes a partir da análise das estratégias relacionadas às empresas estatais tradicionais, às grandes empresas voltadas inicialmente e ainda majoritariamente ao mercado local e às empresas de base tecnológica (quadro 1).

\section{Quadro 1: Sistematização da política industrial e tecnológica chinesa segundo diferentes estratégias, instrumentos, formas de participação do Estado e de internacionalização.}

\begin{tabular}{|c|c|c|c|}
\hline & SOEs tradicionais & $\begin{array}{c}\text { Grandes voltadas ao } \\
\text { mercado local }\end{array}$ & $\begin{array}{c}\text { Empresas de base } \\
\text { tecnológica }\end{array}$ \\
\hline $\begin{array}{c}\text { Diferentes estratégias } \\
\text { tecnológicas }\end{array}$ & $\begin{array}{l}\text { Atualização e } \\
\text { modernização }\end{array}$ & $\begin{array}{c}\text { Catching-up, design e } \\
\text { marcas }\end{array}$ & Fronteira \\
\hline $\begin{array}{c}\text { Diferentes instrumentos } \\
\text { de política industrial e } \\
\text { tecnológica }\end{array}$ & $\begin{array}{l}\text { Licenças e conteúdo } \\
\qquad \text { local }\end{array}$ & $\begin{array}{l}\text { Eficiência dinâmica } \\
\text { (schumpteriana e } \\
\text { keynesiana) }\end{array}$ & $\begin{array}{c}\text { Conhecimento, } \\
\text { financiamento, } \\
\text { padrões tecnológicos e } \\
\text { modelo de negócios } \\
\text { locais }\end{array}$ \\
\hline $\begin{array}{l}\text { Diferentes formas de } \\
\text { participação do Estado }\end{array}$ & SASAC & $\begin{array}{l}\text { Socialização do } \\
\text { financiamento }\end{array}$ & Sistêmica \\
\hline $\begin{array}{l}\text { Diferentes formas de } \\
\text { internacionalização }\end{array}$ & $\begin{array}{l}\text { Tradicional / acesso a } \\
\text { recursos, a mercados, } \\
\text { etc }\end{array}$ & $\begin{array}{c}\text { Aquisição de marcas e } \\
\text { mercados }\end{array}$ & $\begin{array}{c}\text { F\&A, de natureza } \\
\text { tecnológica }\end{array}$ \\
\hline
\end{tabular}

Fonte: elaboração própria, a partir de Nolan (2013 e 2014), Bresser-Pereira et al (2020), Lee (2018), Burlamarqui (2017).

As empresas estatais tradicionais (state-owned enterprises - SOEs) ainda têm no mercado chinês a principal fonte de seu faturamento. Apesar de sua presença destacada na economia, em geral são mais relevantes em setores característicos do paradigma produtivo fordista, como complexo petroquímico, indústrias de base e metal mecânica. Tais empresas tem avançado na estratégia de internacionalização a partir de formas tradicionais, por meio da busca por recursos naturais e acesso a mercados para ampliar seu potencial de acumulação ${ }^{2}$. Dentre elas podem ser citadas as gigantes petroleiras (Sinopec, China National Petroleum, CNOOC), de geração de energia (State Grid, Three

\footnotetext{
${ }^{2}$ Segundo o Ranking 500 Fortune, em 2019, entre as 100 maiores empresas mundiais em receita, havia 24 chinesas. Já entre as 500 maiores, esse número é de 124, face a 121 dos EUA.
} 
Gorges) do setor químico (Chemical China e Sinochem), dos setores de equipamentos de transporte e engenharia (China State Construction Engineering), de alimentos e bebidas (COFCO) entre inúmeras outras.

Como principais diretrizes da atuação da política industrial sobre estas empresas destaca-se a necessidade de se avançar na atualização e modernização produtiva e tecnológica (NOLAN, 2013 e 2014). Para tal, instrumentos tradicionais como exigência de licenças prévias para atuação no mercado chinês, reservas de mercado e exigências de conteúdo nacional são mobilizados. Estes, são potencializados por um amplo esforço de coordenação das decisões de investimento direcionadas a partir da SASAC (JABBOUR e de PAULA, 2020).

No caso das grandes empresas voltadas inicialmente e majoritariamente ao mercado local em setores não coincidentes com aqueles das SOEs tradicionais, o objetivo da política industrial e tecnológica reside na consolidação da estratégia de catching-up, por meio da centralização empresarial, do avanço para atividades mais intensivas em P\&D e da construção de marcas (NOLAN, 2013 e 2014). Dentre tais empresas destacam-se aquelas voltadas ao segmento de bens de consumo duráveis - como a Haier e a Midea -, as inúmeras empresas automobilísticas - estatais ou não, como SAIC, DongFeng, BAIC e BYD -, fabricantes de máquinas e equipamentos - também estatais ou não, como XCMG, LiuGong e Sany - do setor aeronáutico - COMAC - , entre outras.

Para estas empresas o principal desafio parece ser a consolidação do emparelhamento às congêneres estrangeiras, principalmente em atividades com maior capacidade de geração de valor. Para tal, os esforços na construção de suas competências tecnológicas e produtivas, bem como de suas marcas, estão associados a uma estratégia doméstica de fomento em simultâneo da eficiência schumpteriana e keynesiana ${ }^{3}$. A política industrial se ampara no enorme dinamismo do mercado interno para viabilizar a geração de um círculo virtuoso que combine altas taxas de investimento associadas à busca permanente pelo aprendizado tecnológico.

Conforme enfatiza Burlamarqui (2017), este movimento beneficia-se de uma farta disponibilidade de crédito coordenada pelos Big Four bancos chineses (ICBC, CCB, ABC e Bank of China), bem como pelo China Development Bank, pelo Export-Import Bank of China e por bancos regionais. Estes Big Four ocuparam as 3 primeiras e a $5^{\text {a }}$ posição entre os maiores bancos do mundo em termos de ativos em 2019. Segundo dados da Fortune 500 , o total de ativos destes bancos era 57\% maior que a soma dos 4 maiores bancos estadunidenses neste mesmo ano.

Além do apoio financeiro transversal, o tradicional aprendizado derivado do estabelecimento de joint-ventures com empresas transnacionais é potencializado por um sistema de inovação com competências crescentes e recentemente por uma estratégia agressiva de internacionalização em busca da aquisição de marcas e mercados. Dentre casos paradigmáticos para este bloco de empresas, pode-se destacar a compra da sueca Volvo pela Geely, da MG Rover pela SAIC Motors, da Pirelli e da Syngenta pela Chemical China, entre outras.

Por fim, para segmentos nos quais algumas empresas de base tecnológica se situam próximas à fronteira internacional, a participação do Estado é sistêmica e pervasiva, ainda que em muitos casos indireta. Inicialmente, são vultosos e crescentes os recursos disponíveis para o sistema nacional de inovação (ZHOU e LIU, 2016). Segundo dados da OCDE mensurados a partir da paridade de poder de compra, os gastos de P\&D em relação ao PIB chinês evoluíram de 0,89\% em 2000 para 2,4\% em 2018, com uma aceleração do crescimento a partir de meados da primeira década dos anos 2000 (275\% entre 2007 e 2018). Tal fato fez com que em 2018 os gastos em P\&D chineses

\footnotetext{
${ }^{3}$ São conceitos de eficiência que se opõem à perspectiva estática da eficiência alocativa ricardiana ao destacaram a sua dimensão estrutural, seja pela diversificação em direção a setores de maior apropriabilidade e dinamismo inovativo (eficiência schumpeteriana) ou de maior elasticidade-renda e dinamismo de mercado (eficiência keynesiana). Vide Dosi et al (1990).
} 
representassem mais de 95\% dos gastos dos EUA, quase 4 vezes os alemães e 3,2 vezes os gastos japoneses.

Complementarmente, a demanda pública apresenta caráter discricionário em favor de produtos com tecnologias desenvolvidas nacionalmente e novamente o sistema financeiro chinês - bem como o nascente e pujante mercado de venture capital - tem disponibilizado volumes expressivos para a realização doméstica de atividades de P\&D mesmo quando comparados a países líderes tecnologicamente em escala global como EUA, Alemanha e Japão (ZENGLEIN e HOLZMANN, 2019). Tal pujança tem se refletido em diversas dimensões. No que diz respeito apenas às unicorn startups (empresas avaliadas em mais de US\$ 1 bilhão na definição utilizada pelo ranking The Global Unicorn Club da influente plataforma de inteligência de mercado em áreas de alta tecnologia CB Insigths), em julho de 2020 havia 476 unicorn startups no mundo. Destas, 122 eram chinesas e 229 estadunidenses. No entanto, ao se analisar o valor de mercado, as empresas chinesas eram avaliadas em US\$ 312 bilhões, frente a US\$ 316 bilhões das americanas.

Segundo Lee (2018), beneficiando-se deste cenário e ao avançar tecnologicamente de maneira muito rápida, encontram-se uma miríade de empresas majoritariamente voltadas à Economia Digital como Baidu, Alibaba, Tencent, iFlytek, SenseTime, Megvii ,Huawei, ZTE, Cambricon, Lenovo, Xiaomi, Vivo e Oppo (do grupo BBK Eletronics), as fabricantes de chips semicondutores SMIC (Semiconductor Manufacturing International Corp.) e HiSilicon, entre outras. Tais avanços, por sua vez, também podem ser visualizados a partir do rápido e intenso movimento de algumas destas empresas para posições de destaque nos rankings internacionais de patenteamento, o que se repete entre universidades e institutos de P\&D (vide tabela 1).

Tabela 1 - Representantes chineses entre os maiores depositantes de patentes na WIPO (World Intellectual Patent Organization)

\begin{tabular}{|c|c|c|c|c|c|c|c|}
\hline \multirow{2}{*}{$\begin{array}{l}\text { Tipo de } \\
\text { Agente }^{\mathrm{a}}\end{array}$} & \multirow{2}{*}{$\begin{array}{c}\text { Posição no } \\
\text { rank em } \\
2017\end{array}$} & \multirow[b]{2}{*}{ Nome } & \multicolumn{5}{|c|}{ Número de depósitos } \\
\hline & & & 2013 & 2014 & 2015 & 2016 & 2017 \\
\hline \multirow{10}{*}{ Empresas (a) } & & HUAWEI TECHNOLOGIES CO., LTD. & 2.110 & 3.442 & 3.898 & 3.692 & 4.024 \\
\hline & & ZTE CORPORATION & 2.309 & 2.179 & 2.155 & 4.123 & 2.965 \\
\hline & & BOE TECHNOLOGY GROUP CO., LTD & 353 & 553 & 1.227 & 1.673 & 1.818 \\
\hline & 13 & LE HOLDINGS (BEIJING) CO., LTD. & - & - & - & 9 & 1.397 \\
\hline & 18 & $\begin{array}{l}\text { SHENZHEN CHINA STAR OPTOELECTRONICS TECHNOLOGY CO., } \\
\text { LTD }\end{array}$ & 916 & 904 & 710 & 1.163 & 972 \\
\hline & 28 & ALIBABA GROUP HOLDING LIMITED & - & - & 16 & 452 & 707 \\
\hline & 32 & TECENT TECNOLOGY (SHENZHEN) COMPANY LIMITED & 359 & 1.086 & 981 & 172 & 560 \\
\hline & 34 & $\begin{array}{l}\text { YULONG COMPUTER TELECOMMUNICATION SCIENTIFIC } \\
\text { (SHENZHEN) CO., LTD }\end{array}$ & - & - & 71 & 256 & 517 \\
\hline & 40 & GUANGDONG OPPO MOBILE TELECOMMUNICATIONS CORP., LTD & - & - & 27 & 80 & 474 \\
\hline & 49 & XIAOMI INC. & - & - & 126 & 298 & 354 \\
\hline \multirow{10}{*}{$\begin{array}{l}\text { Instituições } \\
\text { de Ensino } \\
\text { Superior (b) }\end{array}$} & $222(11)^{*}$ & SHENZHEN UNIVERSITY & - & - & 29 & 87 & 108 \\
\hline & $244(14)$ & CHINA UNIVERSITY OF MINING AND TECHNOLOGY & - & - & 43 & 84 & 99 \\
\hline & $272(19)$ & TSINGHUA UNIVERSITY & - & - & 120 & 84 & 90 \\
\hline & $337(27)$ & SOUTH CHINA UNIVERSITY OF TECHNOLOGY & - & - & 49 & 50 & 70 \\
\hline & $351(28)$ & PEKING UNIVERSITY & - & - & 81 & 54 & 67 \\
\hline & $362(30)$ & JIANGNAN UNIVERSITY & - & - & 12 & 37 & 65 \\
\hline & $427(35)$ & ZHEJIANG UNIVERSITY & - & - & 28 & 37 & 53 \\
\hline & $444(38)$ & $\begin{array}{l}\text { ZHANGJIAGANG INSTITUTE OF INDUSTRIAL, TECHNOLOGIES } \\
\text { SOOCHOW UNIVERSITY }\end{array}$ & - & - & 18 & 18 & 51 \\
\hline & $459(41)$ & JIANGSU UNIVERSITY & - & - & 9 & 30 & 50 \\
\hline & $481(45)$ & SHANGHAI JIAOTONG UNIVERSITY & - & - & 32 & 30 & 48 \\
\hline \multirow{3}{*}{$\begin{array}{l}\text { Institutos de } \\
\text { Pesquisa do } \\
\text { Governo (c) }\end{array}$} & $106(3)^{* * *}$ & CHINA ACADEMY OF TELECOMMUNICATIONS TECHNOLOGY & - & - & 118 & 145 & 204 \\
\hline & $320(9)$ & SHENZHEN INSTITUTE OF ADVANCED TECHNOLOGY & - & - & 18 & 31 & 75 \\
\hline & $749(27)$ & CHINA ELETRIC POWER RESEARCH INSTITUTE & - & - & 1 & 6 & 32 \\
\hline \multicolumn{3}{|l|}{ Total em a } & 6.047 & 8.164 & 9.211 & 11.918 & 13.788 \\
\hline \multicolumn{3}{|l|}{ Total em b } & - & - & 421 & 511 & 701 \\
\hline \multicolumn{3}{|l|}{ Total em c } & - & - & 137 & 182 & 311 \\
\hline
\end{tabular}

Fonte: SILVA (2019), a partir de WIPO (2015); WIPO (2018).

Para essas empresas, que constituem o ponto nevrálgico da almejada construção da liderança tecnológica global, a estratégia de desenvolvimento empresarial está 
frequentemente entrelaçada à política tecno-nacionalista (NAUGHTON, 2020, MAJEROWICZ e MEDEIROS, 2018, MAJEROWICZ, 2019). Tal fato pode ser observado ativamente nos esforços do Estado chinês para a difusão internacional de padrões tecnológicos definidos localmente, do qual a disputa em torno dos padrões de telecomunicações 5G é o caso mais emblemático (LEE, 2018). Ainda como caso ilustrativo deste movimento, ao analisar a internacionalização do Alibaba na Ásia, Naughton (2020) sugere a emergência de uma Digital Silk Road como vetor da estratégia chinesa na região.

Nestes segmentos o incentivo à internacionalização também se configura como um instrumento para a política industrial e tecnológica fomentar o aprendizado, uma vez que esta congrega esforços simultâneos de busca por novos mercados, aquisições de capacitações tecnológicas e inovativas, bem como de tentativa de internacionalização de padrões tecnológicos nacionais. Assim, esta internacionalização tende a se direcionar prioritariamente a países onde os sistemas nacionais de inovação contribuem para o incremento da competitividade tecnológica local.

A partir do esforço de agregação das estratégias de desenvolvimento produtivo e tecnológico da estrutura produtiva chinesa baseado em três grandes grupos, compreendese que os efeitos destas estratégias na estrutura produtiva doméstica e internacional também serão heterogêneos. Ou seja, serão condicionados pelos estágios específico de desenvolvimento de cada um dos grandes blocos e atores envolvidos. É exatamente neste contexto que devem ser analisados com mais detalhes os limites e as possibilidades da estratégia chinesa de se constituir como uma Superpotência Industrial e da Internet em 1949, exposta com maior ênfase no Made in China 2025.

\section{Os limites à estratégia chinesa de consolidação de uma Superpotência Industrial e da Internet: algumas considerações a partir da heterogeneidade da Indústria 4.0}

De maneira geral, o fomento às tecnologias associadas à Indústria 4.0 reforçam o entrelaçamento sistêmico entre a estrutura produtiva local e os objetivos geopolíticos do Estado Chinês. Por meio de uma abordagem top down de reforço do tecno-nacionalismo, a política industrial tem como eixo central de estruturação dos esforços de longo prazo a construção de 40 centros nacionais de P\&D nas tecnologias core da indústria 4.0.

Uma vez que muitas destas tecnologias ainda se encontram no que Utterback (1996) denomina de estágios fluidos, os esforços iniciais privilegiam iniciativas de experimentação, por meio do estabelecimento de zonas de demonstração tecnológica, criação de projetos pilotos de cidades inteligentes e fábricas verdes e inteligentes. Tanto esses laboratórios quanto as iniciativas pilotos seriam distribuídas espacialmente, de maneira a se aproveitarem das diferentes competências tecnológicas e produtivas regionais. Segundo Zenglein e Holzmann (2019), entre 2015 e 2018, foram lançados 1.646 projetos pilotos em green manufacturing, $854 \mathrm{em}$ smart manufacturing, $388 \mathrm{em}$ manufacturing \& internet integration, entre outros.

Além da ampla rede de institutos de $\mathrm{P} \& \mathrm{D}$ públicos, as empresas constituintes do que Nolan (2001) denominou de National Team, teriam papel fundamental nestes esforços experimentais. A estas, tem sido agregadas uma nova geração de empresas de base tecnológica pujantes em termos tecnológicos e financeiros, com destaque para já citadas Baidu, Alibaba e Tencent (que rivalizariam internacionalmente com as congêneres americanas Google, Amazon e Facebook), além de novos proeminentes agentes em inteligência artificial (iFlytek, SenseTime e Megvii) e em semicondutores (como a HiSilicon, subsidiária da gigante Huawei e a SMIC).

O desenvolvimento tecnológico destas empresas, por sua vez, teria como objetivo reduzir a dependência chinesa por tecnologias centrais ao novo paradigma tecnoeconômico em gestação. Para tal, se beneficiariam de uma quantidade substancial de recursos em uma miríade de programas de fomento direto e indireto, dentre os quais se destacam o National Integrated Circuit Fund (com dotação de 19 bilhões de euros) e o Emerging Industries Investment Funding (5,4 bilhões de euros). 
Além do fomento às atividades de $\mathrm{P} \& \mathrm{D}$ e da implementação de centenas de projetos pilotos domésticos, tais recursos também capitalizam iniciativas de empresas locais ao realizarem aquisições de concorrentes internacionais com o intuito de incorporar capacitações tecnológicas, bem como estabelecer centros de P\&D no exterior. Como exemplo paradigmático desta estratégia cita-se a aquisição por parte da empresa de equipamentos elétricos e eletrodomésticos chinesas MIDEA - que já havia adquirido a fabricante de condicionadores de ar Springer Carrier - da empresa alemã KUKA, especializada no desenvolvimento de robôs industriais e com fortes capacitações tecnológicas na Indústria 4.0.

Ainda vale destacar o caso da SMIC. Esta configura-se como a principal alternativa para contornar as limitações crescentes impostas à venda de chips a empresas chinesas. Apesar de ainda não ser capaz de produzir chips mais avançados tecnologicamente (a SMIC é capaz de produzir unidades de 14nm enquanto que a TMSC produz unidades de $5 \mathrm{~nm}$ ), recebe injeções gigantescas de capital para avançar tecnologicamente. Em maio de 2020 foram aportados ao seu capital US\$ 2,25 bilhões por fundos vinculados a diversas esferas do governo chinês. Já em julho, a empresa recebeu US \$ 6,55 bilhões adicionais por meio de uma oferta inicial de ações na bolsa de Xangai ${ }^{4}$.

Além do incentivo ao aprimoramento tecnológico doméstico e à internacionalização por meio aquisições de tecnologias estratégicas, o financiamento e a redução de incerteza ainda seriam complementados diretamente pela utilização do poder de compra pública e pela abundante disponibilidade de crédito por parte do sistema financeiro doméstico.

Apesar destas diretrizes gerais nortearem as iniciativas com relação ao desenvolvimento das tecnologias associadas à Indústria 4.0, vale destacar que a melhor compreensão da estratégia chinesa exige uma análise mais pormenorizada das principais tecnologias envolvidas da emergência desse novo padrão tecnoprodutivo. A avaliação aqui proposta parte de uma sistematização dessas tecnologias em três camadas distintas, bem como a identificação de tecnologias pervasivas e transversais a essas camadas (figura 1).

\footnotetext{
${ }^{4}$ Segundo relatórios de consultorias especializadas, a SMIC ocupa o quarto lugar em vendas de chips. As três líderes são TSMC (Taiwan), GlobalFoundries (EUA) e United Microelectronics Corporation (Taiwan). Estima-se que a empresa esteja cerca de 5 anos atrasada em relação ao nível de desenvolvimento tecnológico da líder de mercado TSMC, e represente cerca de 10\% da receita desta (WUBBEKE et al, 2016).
} 
Figura 1: Camadas tecnológicas associadas à Indústria 4.0: um esforço de sistematização

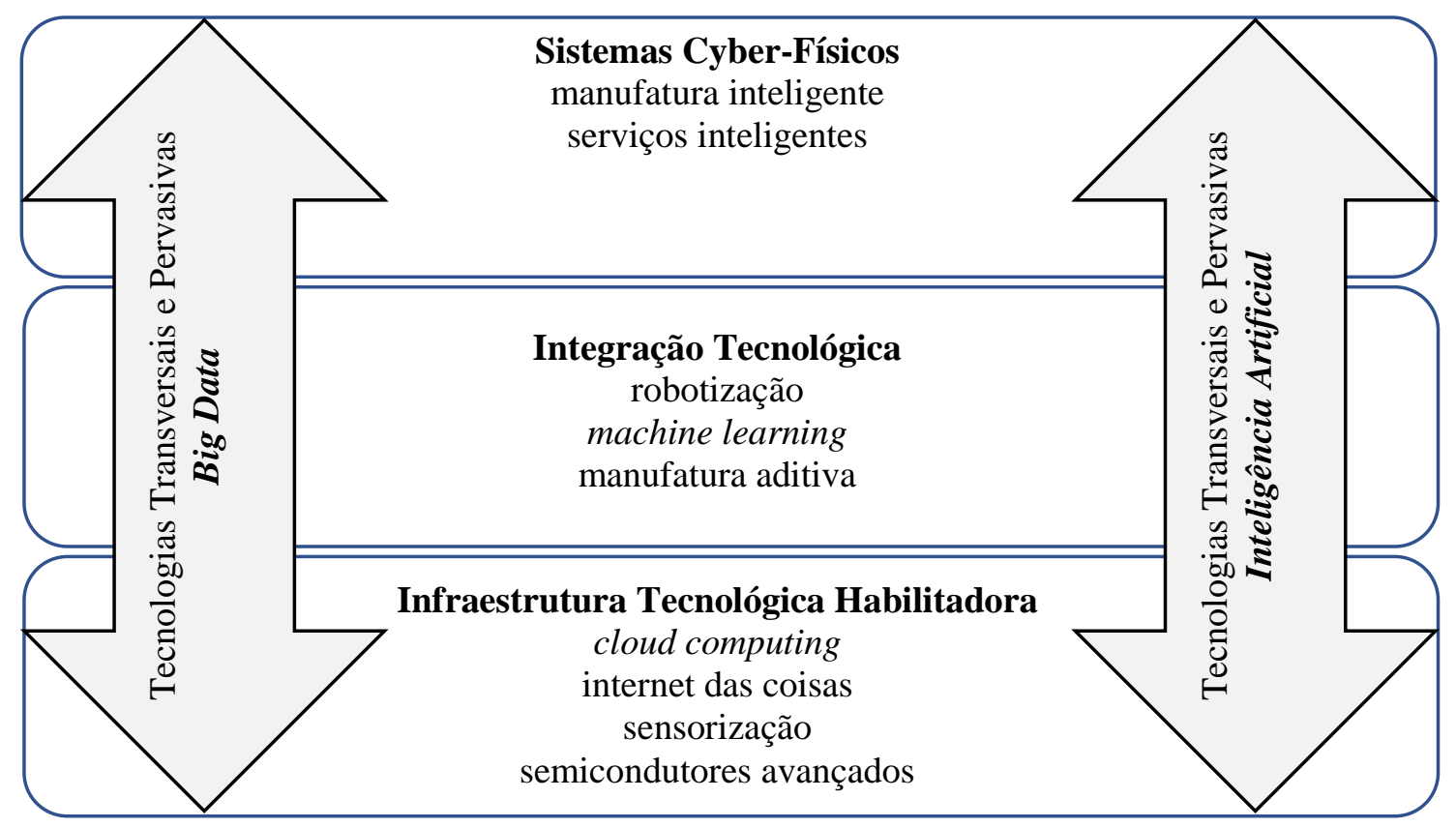

Fonte: elaboração própria, a partir de Schwab (2016 e 2018) e Butollo (2020)

Dentre os componentes deste paradigma, aqueles em que a economia chinesa parece estar mais bem posicionada, é o constituído pelas tecnologias transversais de Inteligência Artificial e Big Data, com a materialização em Sistemas Cyber-Físicos baseados em Serviços Inteligentes, conforme sugerem Lee (2018), Naughton (2020), Zenglein e Holzmann (2019) . Isso porque esta se beneficiaria de vantagens como o enorme mercado doméstico protegido, o acesso a um volume gigantesco de dados, o poder de coordenação pública para implementação de tecnologias em cidades inteligentes e a existência de players locais de destaque internacional, com amplo conhecimento das especificidades do mercado doméstico e beneficiando-se de expressivas externalidades de rede derivadas da amplitude de suas plataformas tecnológicas.

O principal agente destas plataformas na dimensão digital - o WeChat - possuía em 2017 mais de 900 milhões de usuários que utilizam diariamente uma diversidade de serviços integrados à plataforma a partir do crescente avanço de fusão entre sistemas físicos e virtuais. Tal fusão, por sua vez, assume tamanha dimensão que tem viabilizado a explosão do volume movimentado via pagamentos móveis. Estes, segundo as consultorias I-research e McKinsey, alcançaram US\$ 15,4 trilhões em 2017, ou mais de 40 vezes o valor movimentado nos EUA. É nesses segmentos em que se situam a maior parte das empresas de base tecnológicas citadas anteriormente, que tem se destacado internacionalmente.

Outros dois importantes veículos de integração destas tecnologias em Sistemas Cyber-Físicos baseados em Serviços Inteligentes são as iniciativas de City Brain e as Taobao Villages, ambas coordenadas majoritariamente a partir de plataformas tecnológicas comandadas pelo Alibaba. A partir de esforços pioneiros em sua cidade sede (Hangzhou), o grupo tem buscado integrar um conjunto amplo de soluções aptas a

\footnotetext{
${ }^{5}$ Zenglein e Holzmann (2019) citam entre as áreas nas quais o desenvolvimento tecnológico autóctone chinês é mais consolidado veículos autônomos, cidades inteligentes e reconhecimento facial. Em contrapartida, as áreas com maiores lacunas e maior dependência externa são aquelas habilitadoras como semicondutores, novos materiais e relacionadas à pesquisa básica.
} 
viabilizarem a digitalização inteligente de uma miríade de serviços públicos (em sentido lato, não jurídico) a partir do Alibaba Cloud. Com o aperfeiçoamento da plataforma a partir da utilização de inteligência artificial, diversas derivações de políticas são sugeridas no sentido de aumentar a eficiência e o bem estar da população (seja em soluções mais tradicionais como gestão do tráfego, até a utilização de sistemas complexos de rastreamento de disseminação de doenças, combate a seu espraiamento e disponibilidade de soluções para o posterior tratamento por meio do sistema de saúde).

Com a consolidação tecnológica destas soluções, e após seu empacotamento em uma plataforma liderada pelo gigante chinês de tecnologia, a empresa tem se beneficiado da influência chinesa na Ásia para internacionalizar a oferta de tais soluções. Por meio de projetos financiados domesticamente, tais esforços têm se materializado num vetor de transnacionalização dos padrões tecnológicos chineses, via o que se tem convencionado denominar de Digital Silk Road (NAUGHTON, 2020). Assim, coordenados pela iniciativa City Brain, espalhada em 23 cidades pela Ásia, empresas chinesas em tecnologias correlatas se internacionalizam e consolidam suas soluções tecnológicas. Dentre elas pode-se citar as fabricantes equipamentos de vigilância via vídeo Hikvision e Dahua, a fabricante de equipamentos de transmissão de dados New H3C Technologies, entre outras. Vale lembrar, inclusive, que estes esforços iniciais se encontram no bojo de um debate mais amplo por parte da política de desenvolvimento tecnológica chinesa de incentivar o estabelecimento de padrões tecnológicos locais como forma de contornar a dependência por tecnologias externas, por meio de um suposto plano em esboço denominado China Standards 2035 (o qual se insere no contexto das discussões para a elaboração do $14^{\circ}$ Plano Quinquenal).

Esforço semelhante tem sido observado a partir do fomento ainda majoritariamente nacional às Taobao Villages. Estas, também lideradas pelo grupo Alibaba, integram vilas geograficamente dispersas à plataforma de comercialização global da empresa. A lógica é a de oferecer um conjunto de serviços inteligentes às comunidades locais - como ferramentas de gestão de demanda, de previsão de comportamento dos consumidores, de gestão da cadeia de suprimentos, de serviços de marketing e financeiros, entre inúmeros outros - de modo a viabilizar ao mesmo tempo a produção em massa, a baixo custo e com elevado grau de customização. Essa revolução é habilitada pela integração cyber-física baseada em serviços inteligentes que, por meio de inteligência artificial, permite identificar padrões de comportamento dos consumidores e, de maneira preditiva sinalizar determinadas tendências de demanda.

Uma vez que há restrições ao acesso das gigantes globais de serviços (como Google e Facebook, entre outras) ao mercado chinês, e dado o elevado nível de desenvolvimento operacional e tecnológico das empresas locais, os sistemas cyber físicos baseados em serviços inteligentes parecem ser a área da Indústria 4.0 com maior destaque relativo chinês na guerra tecnológica com os EUA. Tal vantagem ainda se potencializa na medida em que a área de influência econômica e política chinesa na Ásia tem sido utilizada como um vetor para internacionalização de padrões tecnológicos locais.

Entretanto, as pressões dos EUA para barrar a internacionalização destes padrões para regiões sobre sua influência, como Europa e América Latina, parece ser um importante fator limitador do sucesso da estratégia de internacionalização chinesa para além do espaço asiático.

Já nos segmentos que estão relacionados diretamente com as atividades manufatureiras como a constituição de sistemas cyber físicos por meio de tecnologias de integração como robotização, machine learning e manufatura aditiva, o posicionamento chinês pode se beneficiar da existência de um forte e diversificado parque produtivo. Além disso, a existência de uma complexa cadeia de suprimento local e do alto poder de coordenação dos investimentos realizados pelas grandes estatais também podem contribuir para iniciativas de desenvolvimento conjunto entre empresas privadas, estatais e centros de P\&D.

Apesar do bom potencial presente nestas parcerias (inclusive algumas delas realizadas sobre a supervisão de instituições de pesquisa alemãs para projetos pilotos, 
como lembram ZENGLEIN e HOLZMANN, 2019), vale lembrar que a estrutura produtiva doméstica ainda é bastante heterogênea. Assim, a despeito da existência de empresas de classe global em diversos setores, as lacunas no índice de informatização e robotização do parque manufatureiro chinês colocam desafios para o sucesso de uma suposta transição para um novo paradigma ${ }^{6}$. Em outras palavras, são inúmeras as deficiências para a universalização no parque produtivo local de tecnologias associadas a $3^{\mathrm{a}}$ Revolução Industrial, o que poderia dificultar no médio prazo a transição almejada para uma suposta $4^{\mathrm{a}}$ Revolução.

Adicionalmente, segundo Butollo (2020), mesmo para as empresas tecnológica e financeiramente superiores, a própria generalização da adoção de tecnologias associadas à indústria 4.0 não pode ser compreendida a partir de uma perspectiva tecnicista. Isso porque fatores como custos de implementação, disponibilidade de financiamento, necessidade de desenvolvimento de competências tecnológicas e gerenciais complementares e lucratividade econômica são variáveis decisivas para a decisão de migração tecnológica, mesmo em um cenário em que as máquinas, equipamentos e sistemas digitais que habilitam a manufatura inteligente estejam disponíveis. São inúmeros os elementos que reforçam o lock-in (técnico, gerencial, de conhecimento e financeiro) aos sistemas de produção e comercialização já integrados às densas redes de fornecedores e compradores.

Uma vez que tais tecnologias podem ser compreendidas como dedicadas a atividades com menor impacto em dimensões sensíveis política e militarmente, o potencial de tensão na guerra tecnológica entre China e EUA parece ser relativamente menor. Apesar da importância do controle dos padrões com vistas a oferecer soluções completas também ser uma variável relevante para a dinâmica da concorrência nestes segmentos, os impactos derivados das políticas tecno-nacionalistas do EUA são mais indiretos. Adicionalmente, com vistas a contornar tais impactos, as iniciativas de política industrial chinesa a partir do Made in China 2025 têm incentivado o estabelecimento de parcerias na área de manufatura inteligente entre agentes locais (estatais e centros de P\&D) e instituições de pesquisa e empresas alemãs. Estas, cientes da relevância do parque produtivo chinês como maior mercado consumidor potencial do mundo, tentam se posicionar - apoiadas pelas políticas nacionais - como "fábricas das fábricas da indústria 4.0". (BUTOLLO, 2020; SCHWAB, 2016 e 2018).

Por fim, na camada de infraestrutura tecnológica habilitadora das fábricas e dos serviços inteligentes (como cloud computing, internet of things e sensorização), novamente empresas como as gigantes de telecomunicações Huawei e ZTE e as de internet Alibaba, Baidu e Tencent têm colocado as iniciativas chinesas em destaque internacional. No entanto, a forte dependência tecnológica de empresas como ASML, TSMC, Qualcomm, Samsung e ARM para a importação de semicondutores de alta performance pode ser um importante obstáculo para o espraiamento na economia de tecnologias habilitadoras como inteligência artificial.

Parece ser exatamente este o ponto mais frágil de toda a estratégia chinesa em busca da liderança nas tecnologias associadas à Indústria 4.0, conforme também destacam Zenglein e Holzmann (2019). Não obstante os enormes esforços para fomentar a construção de capacitações em empresas nacionais de semicondutores como HiSilicon, SMIC e Cambricon, as crescentes restrições impostas pelo governo dos EUA à venda de tecnologias estadunidenses às empresas chinesas podem ser um empecilho difícil de ser transposto pela política industrial chinesa.

A escalada deste movimento tem reverberado nas relações geopolíticas entre um conjunto amplo de países para além dos EUA e tem levado inclusive à adoção de restrições crescentes a inúmeras tentativas de aquisições por empresas chinesas de firmas europeias com atuação em tecnologias estratégicas. Essas negativas invariavelmente têm

\footnotetext{
${ }^{6} \mathrm{Na}$ Coréia do Sul, Japão, Alemanha e EUA a densidade de robôs industriais por 10 mil trabalhadores era em 2015, respectivamente, de mais de 520,300, 300 e cerca de 180. Na China este patamar se situava em torno de 25.
} 
como justificativa o fato de que o avanço chinês colocaria em risco a segurança e soberania nacional dos países sedes destas empresas alvos. Como um dos casos, cita-se a tentativa de aquisição em 2016 - barrada pelo governo alemão - por parte de um fundo de investimento chinês da empresa alemã Aixtron, fabricante de máquinas utilizadas no processo produtivo para a manufatura de chips (MAJEROWICZ e MEDEIROS, 2018, MAJEROWICZ, 2019).

Ainda no que diz respeito à dependência nas camadas mais básicas da infraestrutura tecnológica habilitadora das tecnologias pervasivas da Indústria 4.0, tem ganhado destaque a crescente pressão estadunidense para que empresas fornecedoras de máquinas, equipamentos e tecnologias necessárias para a construção de foundries de semicondutores restrinjam suas vendas a empresas chinesas. $\mathrm{O}$ caso emblemático, que revela a dependência tecnológica chinesa nestas camadas, refere-se às pressões do departamento de Estado para que a holandesa ASML não forneça equipamentos à principal fabricante chinesa de chips semicondutores SMIC.

A ASML é a principal fabricante global de equipamentos avançados de litografia , tidos como necessários para a evolução tecnológica na miniaturização dos circuitos integrados. Em outras palavras, no limite, é a capacidade tecnológica da ASML de desenvolver máquinas cada vez mais precisas que condiciona a evolução da cadeia produtiva dos semicondutores. Ou, palavrassem termos da própria empresa, a capacidade de modificar toda a indústria global de semicondutores "um nanômetro de cada vez". Deste modo, quaisquer restrições da oferta de equipamentos da ASML a fabricantes de semicondutores chineses têm importantes efeitos na capacidade de desenvolvimento das tecnologias habilitadoras da indústria $4 \cdot 0^{7}$.

Conforme lembram Majerowicz e Medeiros (2018) e Majerowicz (2019), é exatamente nos segmentos de semicondutores que reside a maior limitação para o avanço da política industrial tecno-nacionalista chinesa. Essa limitação, a partir de meados de 2020 parece ser virtualmente incontornável no curtíssimo prazo, dado três blocos de restrições impostas direta e indiretamente pelos EUA.

O primeiro diz respeito à limitação imposta indiretamente a qualquer empresa estadunidense ou não - que utilize em alguma etapa do seu processo produtivo tecnologias de origem americana de comercializar seus produtos e serviços com as principais empresas chinesas voltadas ao desenvolvimento das tecnologias da indústria 4.0. Uma vez que as tecnologias estadunidenses são virtualmente onipresentes nos diversos segmentos constituintes da indústria 4.0, tal restrição assume caráter de difícil transposição imediata.

$\mathrm{O}$ segundo bloco de restrições diz respeito a pressões diretas para a empresas chaves na cadeia de suprimento de semicondutores cessarem parcerias com empresas chinesas, dentre os quais os casos mais emblemáticos são o fim da venda de circuitos integrados de última geração da TSMC e a pressão para que a ASML também restrinja vendas de seus equipamentos de última geração a empresas chinesas. Vale mencionar que a oferta global de chips é extremamente concentrada e que a TSMC é a líder no segmento. Destaca-se que a maior parte das empresas de tecnologia que utilizam chips de última geração são fabless, ou seja, são responsáveis apenas pelo design e pela concepção desses chips - os quais são fabricados por terceiros, invariavelmente a taiwanesa TSMC quando se tratam de chips de última geração. Tal arranjo embarca tanto empresas chinesas como Huawei, ZTE, Xiaomi, Hi-Silicon, Cambricon, Vivo, Oppo entre outras, quanto gigantes globais como Apple, Qualcomm, etc. Exceções parciais a esta dinâmica são a coreana Samsung e a estadunidense Intel. É exatamente por estes motivos que, adicionalmente, o governo dos EUA tem atuado fortemente para convencer a taiwanesa TSMC a deslocar parte sensível de sua produção para novas fábricas a serem construídas nos EUA.

O terceiro bloco de restrições refere-se a pressões para que países aliados restrinjam o acesso de empresas e tecnologias chinesas a seus mercados, do qual a batalha

\footnotetext{
${ }^{7}$ A principal fabricante de chips chinesas, a SMIC, é capaz de produzir unidades de $14 \mathrm{~nm}$ enquanto que a TMSC produz unidades de $5 \mathrm{~nm}$, o que representaria um atraso tecnológico da empresa chinesa de cerca de 5 anos.
} 
em torno da definição dos padrões de telecomunicações via $5 \mathrm{G}$ é o principal expoente. Desta forma, além de reduzir o espaço de valorização do capital chinês, a internacionalização de seus padrões tecnológicos seria interditada, pelo menos nestes segmentos.

Como resultado deste cenário e a despeito dos avanços em todas as demais áreas citadas anteriormente, observa-se que a complexidade tecnológica exigida para o desenvolvimento e a fabricação principalmente de chips semicondutores (dentre os quais se incluem aqueles dedicados à inteligência artificial) ainda se configura como a lacuna central da estratégia chinesa expressa no Made in China 2025 e aos objetivos rascunhados para o China Standards 2035. Assim, dado o caráter altamente transversal e pervasivo destes, a superação dessa lacuna se coloca como a pedra angular para viabilizar o espraiamento das demais tecnologias a fim de se forjar um novo paradigma tecnoeconômico que reserve um espaço hierarquicamente superior ao parque produtivo e às tecnologias chinesas.

\section{Considerações Finais}

O acirramento da concorrência intercapitalista e interestatal a partir da crise de 2008 é central para se compreender a retomada da política industrial como um instrumento explícito das estratégias de desenvolvimento nacionais (CHEN \& NAUGHTON, 2016). Além dos potenciais impactos na construção de uma trajetória de crescimento que busque incentivar a eficiência schumpteriana e keynesisana, a reorganização dessas estratégias tem como objetivo central transformar o paradigma tecnoeconômico.

Por meio do incentivo às tecnologias associadas ao que se convencionou denominar de Indústria 4.0, países como EUA e Alemanha têm como objetivo empurrar deliberadamente a fronteira tecnológica e assim reconfigurar os determinantes da dinâmica concorrencial, inovativa e de acumulação (BUTOLLO, 2020). Essa reconfiguração seria um instrumento para reforçarem suas posições de liderança intencional e de alguma maneira se contraporem ao avanço chinês.

Adicionalmente, a Guerra Tecnológica em curso - principalmente no caso dos EUA - é compreendida como um elemento central para a permanente reafirmação de seu domínio geopolítico internacional, dados os desdobramentos das tecnologias da Indústria $4.0 \mathrm{em}$ aspectos militares e de controle e vigilância da sociedade global (MAJEROWICZ e MEDEIROS). Isso porque parece haver uma percepção crescente entre os formuladores de política estadunidenses de que a acoplagem produtiva, tecnológica e financeira sinoamericana nas últimas décadas promoveu condições materiais para o fortalecimento chinês e para sua progressiva afirmação como nação com crescente poder geopolítico internacional. É exatamente nesse contexto que as recentes reações estadunidenses ao avanço chinês devem ser compreendidas.

Entretanto, apesar da proliferação dos sinais do aumento da influência chinesa em diversas dimensões, os limites de sua estratégia devem ser compreendidos criticamente a partir de uma perspectiva mais ampla. A despeito do avanço tecnológico relativamente generalizado, algumas considerações são centrais para delimitar o potencial de enfrentamento à liderança geopolítica internacional dos EUA. Primeiro, a estrutura produtiva chinesa é marcada por um elevado grau de heterogeneidade, o que impõe limites importantes para a disseminação pervasiva das tecnologias da Indústria $4.0 \mathrm{em}$ amplos espectros do tecido produtivo no médio prazo (WUBBEKE el al, 2016; ZENGLEIN \& HOLZMANN, 2019). Segundo, nas principais camadas habilitadoras destas tecnologias - como chips semicondutores de última geração e principalmente o desenvolvimento de máquinas e equipamentos necessários para a produção destes chips - se observa um controle direto e indireto quase onipresente das tecnologias por parte de empresas e instituições de pesquisa dos EUA, as quais muitas vezes possuem ligações históricas com o complexo de defesa (MAJEROWICZ, 2019).

Por fim, apesar desta evidente assimetria em favor do poderio político e tecnológico dos EUA, um fator de influência que não pode ser negligenciado nesta Guerra Tecnológica é a heterogeneidade dos interesses políticos e econômicos internos neste país. 
Assim, um ponto a ser mais bem avaliado com o desenrolar deste movimento estratégico é a posição do grande capital estadunidense já acoplado há décadas à economia chinesa, e que se beneficia amplamente do dinamismo deste país para viabilizar sua valorização em escala ampliada no curto prazo. Em outros termos, a dependência do dinamismo do mercado chinês por parte de grandes empresas de tecnologia dos EUA também é uma variável não desprezível para se compreender até que ponto e por quais caminhos avançará a escalada da Guerra Tecnológica e a desacoplagem da economia sinoamericana.

\section{Referências bibliográficas:}

AKAMATSU, K. (1962) "A historical pattern of economic growth in developing countries", Journal of Developing Economies 1(1) Mar-Aug: 3-25.

BRESSER-PEREIRA, L. C.; JABBOUR, E. e PAULA, L.F. (2020). O catching-up da Coreia do Sul e da China: uma análise novo-desenvolvimentista. Brazilian Journal of Political Economy, 40(2), 264-284. BURLAMARQUI, L. (2017). Schumpeter goes to China: entrepreneurial state, socialization of investment and creative destruction management, TD - UERJ.

BUTOLLO, F. (2020). Digitalization and the geographies of production: Towards reshoring or global fragmentation? Competition \& Change. 1-20.

CHEN, L; NAUGHTON, B. (2016). An institutionalized policy-making mechanism: China's return to techno-industrial policy, Research Policy 45 (10), 2138-2152;

DOSI, G., PAVITT, K., \& SOETE, L. (1990). The economics of technical change and international trade. LEM Book Series.

JABBOUR, E.; de PAULA, L.F. (2020). Socialization of Investment and Institutional Changes in China: A Heterodoxy Approach, Forum for Social Economics, Routledge.

LEE, KAI-FU. (2018). AI superpower - China, Sillicon Valley and the new world order, Houghton Mifflin Harcourt, Boston.

MAJEROWICZ, E. e MEDEIROS, C. A (2018). A POLÍTICA INDUSTRIAL CHINESA NA GEOPOLÍTICA DA ERA DA INFORMAÇÃO: O CASO DOS SEMICONDUTORES. Revista De Economia Contemporânea, Vol.22, n.1.

MAJEROWICZ, E. (2019). A China e a Economia Política Internacional das Tecnologias da Informação e Comunicação, TD 001, UFRN.

NAUGHTON, B. (2019). Economic Reform and Structural Change: The Chinese Experience in The Oxford Handbook of Structural Transformation.

NAUGHTON, B. (2020) Chinese Industrial Policy and the Digital Silk Road The Case of Alibaba, in Malaysia, Asia Policy, Volume 15, Number 1, Jan, pp. 23-39.

NOLAN, P. (2001). China and the Global Economy: National Champions, Industrial Policy, and the Big Business Revolution. New York: Palgrave.

NOLAN, P. (2013). Is China Buying the World?, Polity Press; 1 ed.

NOLAN, P. (2014) Globalization and Industrial Policy: The Case of China, The World Economy Volume 37, Issue 6, June.

PALMA, J.G.. (2009). Flying Geese and Waddling Ducks: The Different Capabilities of East Asia and Latin America to "Demand-Adapt" and "Supply-Upgrade" their Export Productive Capacity. In: STIGLITZ, J.E., CIMOLI, M., DOSI, G. (Eds.). Industrial Policy in Developing Countries. Oxford: Oxford University Press.

SCHWAB, K. (2016). A quarta revolução industrial, São Paulo: Edipro.

SCHWAB, K. (2018). Aplicando a quarta revolução industrial, São Paulo: Edipro.

SILVA, S.T. (2019) A tecnologia como vetor e bússola no processo de desenvolvimento chinês. Tese de Doutorado, IE Unicamp.

UTTERBACK, J. (1996). Dominando a dinâmica da inovação. Rio de Janeiro: Qualitymark.

Zhou, Yu; Liu, Xielin (2016); Evolution of Chinese State Policies on Innovation in Yu Zhou, William Lazonick, and Yifei Sun, eds. China as an Innovation Nation.

ZENGLEIN, M.; HOLZMANN. (2019), A. Evolving Made in China 2025. Merics, July. 
WUBBEKE, J; MEISSNER, M; ZENGLEIN, M; IVES, J; CONRAD, B. (2016). Made in china 2025: The making of a high-tech superpower and consequences for industrial countries. Merics, Dec. 